\title{
Linx
}

Revue des linguistes de l'université Paris X Nanterre

$72 \mid 2015$

Former à l'écrit universitaire, un terrain pour la linguistique?

\section{Ecrits de transition : rapporter et évaluer les propos d'un auteur dans des fiches de lecture}

\section{Nathalie Gettliffe}

\section{OpenEdition}

\section{Journals}

Édition électronique

URL : http://journals.openedition.org/linx/1669

DOI : $10.4000 /$ linx.1669

ISSN : 2118-9692

Éditeur

Presses universitaires de Paris Nanterre

Édition imprimée

Date de publication : 1 septembre 2015

Pagination : 213-232

ISSN : 0246-8743

Référence électronique

Nathalie Gettliffe, «Ecrits de transition : rapporter et évaluer les propos d'un auteur dans des fiches de lecture », Linx [En ligne], 72 | 2015, mis en ligne le 01 mars 2016, consulté le 30 avril 2019. URL : http:// journals.openedition.org/linx/1669; DOI : 10.4000/linx.1669 


\title{
Ecrits de transition: rapporter et évaluer les propos d'un auteur dans des fiches de lecture
}

\author{
Nathalie GETTLIFFE \\ Laboratoire Linguistique, Langue et Parole, Université de Strasbourg, France
}

\begin{abstract}
Résumé
Notre étude se centre sur des écrits de transition (fiches de lecture) produits par des étudiants d'un Master en didactique des langues $(n=26)$. Les recherches antérieures ont mis l'accent sur les écrits longs (rapport de stage, mémoires) et nous pensons qu'il est important de comprendre comment les étudiants d'une discipline qui n'est proposée qu'à partir du niveau Master rapportent les propos d'auteurs et comment ils se positionnent par rapport à ces derniers. En effet, la fiche de lecture est entrevue ici comme un écrit pédagogisé dans un parcours de recherche articulé qui mènera l'étudiant étape par étape à la rédaction de son mémoire. Les fiches de lecture ont été analysées en nous appuyant sur des catégories déjà établies par des analyses antérieures, telles que l'utilisation de la citation, la reformulation, le positionnement énonciatif et le développement d'un point de vue. Au final, la fiche de lecture est révélatrice de difficultés déjà relevées par des recherches portant sur les écrits longs de recherche en formation. La modification des consignes autour de cet écrit ainsi qu'un accompagnement méthodologique plus centré sur les pratiques citationnelles devraient permettre de mieux former les étudiants dans ce parcours de formation à la recherche.
\end{abstract}

Mots-clés

Littéracies universitaires, écrits de transition, fiches de lecture, pratiques citationnelles, positionnement, dispositif de formation à la recherche.

\section{Introduction}

Les écrits universitaires commencent à être étudiés de manière systématique en France depuis une quinzaine d'années et des propositions didactiques se précisent dans différents dispositifs d'accompagnement qui voient peu à peu le jour. Cependant, les variations entre disciplines et types d'écrits exigés dans les différents cycles universitaires (Blaser et Erpelding-Dupuis, 2010) rendent difficiles toute analyse systémique des productions. Les remédiations proposées doivent donc s'appuyer nécessairement sur une analyse fine des discours qui circulent en milieu universitaire et qui sont exigés des étudiants (voir Pollet, 2001 ; Mangiante et Parpette, 2004 ; Mangiante et Parpette, 2011). Parmi ces discours, les écrits longs (mémoire de master, thèse de doctorat) semblent avoir retenu l'attention de nombreux chercheurs car ces «écrits de recherche en formation » (Reuter, 2004) sont au cœur des productions universitaires (pré- et postdoctorales), n'ont pas d'équivalent dans les cycles antérieurs et nécessitent une véritable acculturation aux discours universitaires (Pollet, 2001). Les écrits de «débutants » sont souvent comparés à des articles scientifiques d'experts (par ex. Boch et Grossman, 2002) afin de faire ressortir une des complexités majeure des écrits universitaires, à savoir la polyphonie énonciative qui doit permettre de faire dialoguer l'auteur du travail avec les données de terrain et les théoriciens (Guigue, 1998). Pour notre part, nous pensons qu'il est important de comprendre quelles sont les pratiques des étudiants dans des écrits plus courts afin de mieux saisir la construction des écrits de recherche en formation plus longs. C'est pourquoi nous nous proposons 
de nous centrer, dans cette étude, sur les stratégies mobilisées par des étudiants de Master dans des écrits de transition ${ }^{1}$ (fiches de lecture) afin de mieux cerner leurs pratiques citationnelles et énonciatives pour aménager le dispositif d'accompagnement de formation à la recherche.

\section{Contexte}

\subsection{Dispositif global}

Le master de Didactique des langues et de Français langue étrangère et seconde (FLES) de l'Université de Strasbourg propose un parcours de formation à la recherche comprenant des cours visant à préparer les étudiants à la rédaction de leur mémoire professionnel ou de recherche. Le premier cours, intitulé Techniques documentaires (12h) et proposé au premier semestre, est suivi d'un cours nommé Problématique et revue de la littérature (12h) qui permet aux étudiants de commencer à rédiger les premiers chapitres de leur mémoire. Lors du troisième semestre, les étudiants sont obligés dans le cadre d'une Unité d'Enseignement baptisée Méthodologie de suivre trois cours : Techniques de rédaction (12h), Méthodes qualitatives (12h) et Méthodes quantitatives (12h). Enfin, des rencontres avec le directeur de recherche sont imposées pendant ce même semestre afin de définir avec précision l'objet de recherche, les concepts-clés, la méthodologie de recherche et la structure du mémoire : il s'agit de mobiliser toutes les compétences acquises dans les cours de formation à la recherche antérieurs et de les mettre en pratique individuellement sur un objet d'étude pertinent. Le semestre 4 laisse place à un suivi personnalisé centré sur la collecte de données et la rédaction du mémoire dans sa version finale. Etant donné que la cohorte d'étudiants de Master en didactique des langues est aussi composée de non-francophones, un cours de Français sur Objectifs Universitaires (FOU) (48h) est proposé à ceux qui ont besoin de soutien pour suivre les enseignements du master Didactique des langues et de FLES et pour s'approprier les discours universitaires écrits et oraux qui circulent au sein de cette formation. Au total, ce sont plus de 60 heures de cours en formation à la recherche qui sont proposées aux étudiants francophones inscrits dans le parcours Didactique des langues; les non-francophones bénéficient de plus de 100 heures de cours dans le même domaine.

Notre étude porte plus précisément sur le premier module d'enseignement Techniques documentaires. Ce cours propose aux étudiants non seulement des outils à utiliser pour élaborer une bibliographie sur un thème de leur choix mais développe aussi des compétences en lecture critique par le biais d'exercices pratiques. A titre d'information, sont présentées les thématiques suivantes : esquisser un projet de recherche, se repérer en bibliothèque, mener une recherche sur Internet, se constituer une bibliographie (normes bibliographiques), réaliser une fiche de lecture, éviter le plagiat. L'évaluation finale porte sur la rédaction de 5 fiches de lecture (savoir-faire) et sur un examen sur table (savoirs). Les fiches de lecture serviront ensuite aux étudiants à rédiger au semestre 2 une problématique autour de leur sujet d'étude.

\subsection{Fiches de lecture}

\footnotetext{
${ }^{1}$ Les écrits de transition représentent des productions rédigées avec des contraintes d'écriture dans un but de formation aux écrits de recherche. On peut nommer les fiches de lecture, les problématiques «pédagogiques », les pré-projets, les travaux d'étude et de recherche, les transcriptions et les ébauches d'analyse de données, etc. Pour nous, tous ces écrits devraient normalement avoir une visée pédagogique avec comme objectif de former les étudiants à certaines pratiques scripturales typiques des écrits universitaires de recherche (citation, positionnement énonciatif, formulation de questions de recherche, etc.).
} 
Pour la plupart des étudiants, l'élaboration de fiches de lecture n'est pas un exercice nouveau. En effet, au cours des études primaires et secondaires, des fiches de lecture autour d'œuvres littéraires sont régulièrement exigées par les enseignants de français. Dans le cadre des révisions du baccalauréat de français, de nombreux ouvrages et sites Internet proposent des fiches de lecture qui articulent une présentation de l'œuvre, un résumé de cette dernière, une analyse des personnages principaux et des axes de lectures possibles. Cet archétype de la fiche de lecture reste ancré dans les pratiques universitaires en langues et lettres modernes. Dans les parcours en sciences humaines, les étudiants peuvent aussi avoir été amenés à rédiger des fiches de lecture avec des pratiques variées (notes de lecture, résumé synthétique, critique du document ou une combinaison de plusieurs de ces composantes). En sciences expérimentales, des modèles de fiches de lecture pour des articles sont répertoriées selon le type de recherches scientifiques menées (essai randomisé, étude de cohorte, étude transversale, etc.) et servent à résumer les résultats des expériences afin de pouvoir les comparer. Bref, les pratiques étant variées selon les disciplines, une partie du cours Techniques documentaires porte sur la forme et le contenu d'une fiche de lecture dans le cadre du parcours de formation à la recherche en didactique des langues.

Il paraissait important, dans le cadre de notre discipline qui s'inscrit dans le champ des recherches en sciences humaines, d'utiliser la fiche de lecture comme un exercice essentiel pour faire émerger les concepts-clés d'une thématique ${ }^{2}$ et pour que l'étudiant appréhende la variation (et les contradictions) dans les définitions. Celui-ci a souvent l'impression que les définitions sont monolithiques et qu'elles ne peuvent être questionnées, ce qui va à l'encontre d'une revue de la littérature, exercice-clé dans la rédaction du mémoire de recherche

En proposant un modèle de fiche de lecture (voir annexe 1) qui dissocie le résumé et le commentaire, nous voulions aider les étudiants à mieux comprendre le va-et-vient entre deux postures (rapporter/évaluer des propos) qui leur sera demandé dans une revue de la littérature (semestre 2/mémoire) et aussi à mieux tisser ce même texte en respectant les règles de la polyphonie énonciative. En effet, malgré le parcours de recherche mis en place depuis quelques années, des maladresses subsistent dans les mémoires des étudiants. Par cette procédure en deux temps, rapporter des propos puis évaluer des propos, notre hypothèse est que les étudiants sont mieux à même de poser leur voix en regard de celle de l'auteur (Rinck, 2006) et d'éviter des problèmes de sous-énonciation (Rabatel, 2002). Après une première partie générale reprenant les références du document, l'étudiant est amené à résumer le document choisi en mettant l'accent sur les thèmes et les concepts clés, en utilisant s'il le souhaite des citations et en s'appuyant sur ses propres mots en dehors des citations. La deuxième partie de la fiche appelée commentaires consiste à donner ses impressions, questionner le texte (point de vue) et à énoncer la pertinence du document au vu du thème de recherche. Chaque étudiant rédige 5 fiches de lecture qui sont notées en fin de semestre ${ }^{3}$.

\section{Méthodologie}

\subsection{Recueil de données}

\footnotetext{
${ }^{2}$ Par exemple, quels sont les concepts-clés de la thématique « Enseignement précoce des langues »?

${ }^{3}$ Les étudiants sont aussi invités à s'exercer à réaliser une fiche de lecture et à mettre leur travail en ligne dans une base de données visible par tous. L'enseignant poste ensuite des commentaires consultables par tous les étudiants et propose une fiche modèle réalisée par un étudiant (voir annexe 2).
} 
Notre analyse se centrera sur 26 fiches de lecture parmi les 130 recueillies ${ }^{4}$. Pour chaque étudiant, nous avons choisi une fiche parmi les cinq qu'il devait fournir en privilégiant celle qui était le plus en rapport avec le thème de recherche choisi. En effet, dans chacun des cas, cette fiche de lecture apparaissait comme la plus développée. Sur les 26 fiches de lecture, 12 portent sur des monographies, 4 sur des chapitres de livre, 9 sur des articles scientifiques et 1 sur des actes de colloque. Elles ont été rédigées par 16 locuteurs francophones et 10 locuteurs non-francophones et ont été rendues en novembre 2012.

\section{2 Analyse des données}

Notre étude s'est focalisée sur les différentes stratégies utilisées par les étudiants pour rapporter et évaluer les propos d'un auteur. Etant donné que nous étions dans le cadre d'écrits de transition, il n'était pas d'actualité de travailler sur des erreurs ou des dysfonctionnements comme cela est régulièrement rapporté dans les recherches en littéracies universitaires (Reuter, 2004). Il s'agissait plutôt d'observer les stratégies mobilisées pour tenter de comprendre les problèmes d'écriture polyphonique qui existaient au début de ce parcours de recherche. Comme il existe peu de recherches sur les écrits de transition où le scripteur se construit comme acteur et constructeur de connaissance (Delcambre et Reuter, 2002 :22), nous avons élaboré notre propre grille d'analyse (voir annexe 3) pour chacune des parties de la fiche de lecture.

Pour le résumé, il s'agissait d'observer les stratégies d'effacement énonciatif (non-utilisation du je ; formules impersonnelles ; utilisation du passif), les stratégies pour les citations (nombre de guillemets/citations ; îlot citationnel/citation autonome ; reformulation ; copier/coller), les traces de présentation de l'auteur et/ou du support et l'expression éventuelle d'un point de vue. Pour la partie commentaires, nous avons recensé les occurrences du «je » prenant clairement position, les énoncés évoquant des impressions (j'aime/j'aime pas; pertinence) ou questionnant le texte (marqueurs d'accord et de désaccord ; confrontation avec d'autres textes). Finalement, nous avons décidé aussi de regarder les articulations éventuelles entre les deux parties (transition/formatage ; connecteurs ; reprises d'énoncés).

\section{Analyse de la partie résumé}

Nous présenterons ici les résultats de nos analyses en incluant la discussion dans chaque souspartie.

\subsection{Catégorisation}

Pour recenser les pratiques, nous nous sommes ensuite appuyée sur les catégories de discours rapporté définies par Boch et Grossmann $(2002: 43)^{5}$, à savoir la reformulation, l'îlot citationnel et la citation autonome :

«On peut distinguer trois catégories de DR [discours rapporté]. La citation crée un espace autonome au plan énonciatif, tandis que la reformulation permet au scripteur d'intégrer la

\footnotetext{
${ }^{4}$ Cette première approche est généralisable à l'ensemble des fiches de lecture.

${ }^{5}$ Nous n'avons pas jugé utile de catégoriser les citations de manière plus fine comme a pu le faire Kara (2004) car les stratégies utilisées par les étudiants ne sont pas étendues.
} 
parole de l'autre dans son propre dire, en l'assumant énonciativement. L'îlot citationnel permet à la fois l'intégration et la mise en évidence du segment cité par le marquage scriptural, grâce aux italiques et aux guillemets. »

La citation autonome est définie, quant à elle, comme du discours direct sans intégration syntaxique et comportant un segment introducteur typique « $\mathrm{X}$ a dit : ».

\subsubsection{Citations autonomes}

Nous avons recensé 27 citations autonomes dans 12 copies. Les citations autonomes sont intégrées dans le texte mais elles peuvent aussi apparaître à la fin de la partie Résumé pour faire état de notions ou de définitions-clés, comme par exemple ${ }^{6}$ dans l'exemple suivant ${ }^{7}:$ La pédagogie interculturelle est aussi définie comme un instrument pour promouvoir l'égalisation des chances et une insertion optimale dans la vie économique et sociale (p.87). Les pages sont toujours indiquées dans ce contexte-là. Nous avons aussi identifié un pavé citationnel de 158 mots qui reprend des débats autour d'une notion et qui ressemble à une prise de notes brute. L'étudiant l'ayant rédigé étant étranger, peut-être s'agit-il ici d'une mauvaise compréhension du format du travail.

Pour ce qui est de la longueur, le nombre moyen de mots par citation autonome est de 26. Concernant leur forme, les citations autonomes suivent des verbes introducteurs classiques (dire, affirmer, déclarer). La plupart du temps la ponctuation «: (deux points) est mobilisée. Finalement, la moitié des citations autonomes n'ont pas de pages associées. Par exemple, nous trouverons: Sa conclusion est la suivante: «ces analogies étayent l'idée que le continuum de développement des créoles et les variétés pré-basiques mises en ouvre lors de l'appropriation d'une langue étrangère sont, toutes choses égales d'ailleurs, identiques».

\subsubsection{Ilots citationnels}

Concernant maintenant les ilots citationnels, à savoir des citations intégrées syntaxiquement dans la phrase, elles sont au nombre de 14 dans 4 fiches de lecture. La longueur moyenne des îlots est de 15 mots. Ces îlots servent dans certains cas à terminer une reformulation pour ajouter de l'authenticité (Plusieurs conventions non-dites fonctionnent comme la règle " une langue une personne ${ }^{8} »$; Notons cependant que les « stratégies temporelles échouent la plupart du temps ») mais on peut se demander légitimement s'il ne s'agit pas simplement d'un maquillage. Ils peuvent aussi être annoncés par un double formatage (guillemets et italique).

Au total, il existe très peu de citations définitionnelles (Kara, 2004: 135) ${ }^{9}$. L'étudiant utilise la citation (îlots citationnels et citations autonomes) pour montrer qu'il a lu mais les citations ne sont

\footnotetext{
${ }^{6}$ Les propos des étudiants dans les fiches de lecture seront repris en italique pour les distinguer des propos de chercheurs qui seront présentés entre guillemets.

${ }^{7}$ Les étudiants ont créé dans ce cas un espace séparé entre le Résumé et le Commentaire pour faire ressortir des citations qu'ils considèrent comme importantes.

${ }^{8}$ Cette citation de l'étudiant reprend sous sa forme lapidaire le principe de Ronjat (1913) qui propose que pour optimiser l'apprentissage précoce d'une langue chez l'enfant chaque parent doit se cantonner à parler sa langue première.

9 « Recours à la citation pour fixer les contours d'une notion ou d'un concept».
} 
pas définitionnelles dans un travail dialogique autour des concepts : la faible longueur de la citation ne permettra pas ensuite de comparer différentes définitions d'un même concept.

\subsubsection{Expressions entre guillemets}

Nous avons trouvé beaucoup d'expressions entre guillemets (54 dans 14 fiches de lecture pour une moyenne de 3,7 expressions par fiche de lecture). Nombre de ces passages entre guillemets ne servent qu'à encadrer un mot ou quelques mots mais ne représentent pas des citations à proprement parler. D'ailleurs, seuls 4 étaient accompagnés d'une pagination. Il s'agit d'expressions communes («dépassement de soi », «bon jeu», «grammaire», etc.) qui ne nécessitent a priori pas de guillemets. D'autres suites de mots sont entre guillemets alors qu'elles seraient aussi bien en italiques ou avec une majuscule pour chaque début de mot (Programme «langue vivante »). Les guillemets sont aussi utilisés pour l'insertion de mots étrangers dans le résumé (« Motivational self-regulation»; «integrativeness »; «ideal self», etc.). Enfin, les guillemets entourent aussi des expressions propres au domaine de la didactique des langues mais qui ne sont pas à relier spécifiquement à l'auteur du document rédigé (représentation « subconsciente »des sons; « interlangue » (p.222); «bilinguisme familial »; « dénativisation »; « éveil aux langues », etc.).

Plusieurs explications peuvent être avancées pour comprendre cette «manie » du guillemet. Il est vrai qu'en français la fonction du guillemet est multiple ${ }^{10}$ : celui-ci peut servir à rapporter des propos (discours direct, citation), prendre de la distance par rapport à des propos ou une terminologie avec lesquels le scripteur n'est pas en accord, mettre un terme en exergue (ce procédé est alors en concurrence avec l'italique ou le souligné) ou encadrer un terme étranger. Il est clair que dans certains cas les étudiants utilisent les guillemets au lieu de mettre le mot en italique. Il est aussi évident que par prudence et pour ne pas se faire reprocher de plagier, les étudiants mettent aussi entre guillemets des expressions communes dans la discipline. Une simple consultation des dictionnaires de spécialité (Cuq, 2003 : Robert, 2008) en didactique des langues et de quelques index de manuels de base (Cuq et Gruca, 2002 ; Bertocchini et Costanzo, 2008 ; Tardieu, 2008) permettrait de lever ces ambiguïtés de paternité concept-auteur.

Une fois présenté l'aspect formel de la mise entre guillemets d'énoncés, reste encore à expliquer la fonction de la citation. Selon Reuter (2001 :18), le discours d'autrui peut s'envisager selon deux angles :

« Le premier se structure autour de l'opposition entre ostention (les discours d'autrui sont montrés, tels une collection de pièces dans une vitrine, attestant d'un travail accompli) et fonctionnalisation (les discours théoriques d'autrui sont intégrés, à de multiples endroits et de manière fonctionnelle dans le discours de recherche : ils participent de la construction des questions, de l'étayage des hypothèses, du recueil et du traitement des données, de leur interprétation et de leur mise en relation avec l'état des lieux théoriques...). »

Comme nous l'avons énoncé dans nos propos liminaires, la première partie de la fiche de lecture devait permettre à l'étudiant de définir des concepts ou des problématiques clés. Cependant, la partie Résumé des fiches de lecture fait peu état de ce premier niveau de

\footnotetext{
${ }^{10}$ Pour une présentation des valeurs sémantiques des guillemets, voir Rinck et Tutin (2007)
} 
fonctionnalisation de la citation, à savoir la fonction définitionnelle (Kara, 2004). Comment peut-on alors dans la partie commentaires de la fiche de lecture et encore plus dans une Revue de la littérature construite faire dialoguer des concepts ou des prises de position quand tout retour aux sources semble impossible du fait du manque de pagination? Ce défaut de notation des sources, déjà relevé par Pollet et Piette $(2002: 173)$ dans une étude sur des fiches de lecture d'étudiants en histoire et par Rinck (2006) dans une recherche sur les parties théoriques de rapports de stage d'étudiants de Licence semble être une constante dans les écrits de recherche en formation quel que soit le type d'écrits étudiés.

\subsection{Reformulation}

Selon Boch et Grossman (2002 :44), la reformulation possède «les marques introductives du discours rapporté (Selon..., d'après, pour..., comme X prétend, affirme, etc.), pas de marques scripturales de type guillemet (ou verbale du type, je cite, pour reprendre les termes de...) [et] le discours d'autrui est intégré dans le discours propre et n'a pas d'autonomie énonciative ».

Si l'on regarde maintenant de plus près les stratégies mobilisées par les étudiants pour reformuler les propos d'un auteur, la première et la plus prégnante stratégie est celle de l'utilisation de la structure du type L'auteur dit que... que l'on retrouve dans 18 fiches de lecture. Cette formule se décline en L'auteur introduit, soutient, distingue, présente, démontre, propose, lance, aborde, offre... ou dans son emploi métonymique (Tutin, 2010) « Ce livre propose, indique... ». Certains étudiants n'hésitent pas non plus à utiliser le «on» pour pointer ce que dit l'auteur de son domaine comme dans les exemples suivants : quand on parle de bilinguisme; on nous présente; on nous l'explique... ${ }^{11}$. Les tournures impersonnelles sont aussi présentes dans 10 fiches de lecture, comme Il est fait mention, il existe, il s'agit de... Au total, 23 fiches de lecture (90\%) s'appuient sur deux stratégies : les tournures l'auteur dit que et les tournures impersonnelles pour reformuler les dires d'un auteur. Une seule fiche de lecture s'appuie sur l'utilisation du passif pour rapporter les propos de l'auteur (une autre distinction est faite) et une seule calque la voix de l'auteur en n'utilisant aucune des structures précédemment citées. De manière générale, on peut repérer dès les premières lignes du résumé la stratégie que l'étudiant a choisie pour rapporter les propos de l'auteur et qu'il va maintenir tout au long de la fiche de lecture.

Pour conclure ces deux premières parties sur le discours rapporté dans les fiches de lecture que nous avons analysées, la citation est une stratégie mineure pour rapporter les propos des auteurs : en écartant les expressions entre guillemets, on ne comptabilise que 35 citations pour 26 fiches de lecture (citations autonomes et îlots citationnels). La reformulation reste la stratégie majoritairement utilisée pour rapporter les propos d'un auteur avec 168 instances. Ce résultat ne correspond pas aux conclusions de l'étude menée par Boch et Grossman (2002) sur les différences entre discours de néophytes et discours d'experts où la citation restait la stratégie mobilisée majoritairement par les étudiants lors de la rédaction de rapports de stage $(71 \%$ du discours rapporté) alors que les experts s'appuyaient plus sur la reformulation dans la rédaction de leurs articles de recherche (35\% du discours rapporté). Ceci pourrait s'expliquer par la consigne de départ pour la rédaction de la fiche de lecture («utiliser vos propres mots ») mais aussi par le fait

\footnotetext{
${ }^{11}$ Nous voyons d'ailleurs la synonymie entre les tournures dans les exemples «Il nous explique » et «on nous l'explique » attestés dans les fiches de lecture.
} 
que le genre «fiche de lecture » serait plus perçu comme un exercice de reformulation que de citation dans l'inconscient des étudiants.

\subsection{Effacement/brouillage énonciatif}

Si aucune occurrence de « je »n'apparait dans la partie résumé, 10 fiches de lecture présentent des cas de «nous /on » qui peuvent s'apparenter au « je » et qui participent au brouillage énonciatif (Tutin, 2010 ; Fløttum et Vold, 2010 ; Reutner, 2010). L'effacement du scripteur n'est donc pas total. Ainsi, les étudiants utilisent le «nous » pour maquiller un «je » dans les exemples suivants : Nous allons donner l'exemple cité dans l'ouvrage ; Nous avons constaté ; Notons. Le «nous » est aussi convoqué par les étudiants qui se mettent en scène comme destinataires du discours : Il nous explique; N. Guichon nous propose; On nous présente; Les ethnologues nous l'ont appris. L'utilisation du pronom «on » ajoute un trait «indéfini » (Fløttum et al., 2007) et permet la participation au discours collectif (Delamotte Legrand, 200é : 35). Dans les exemples suivants, il est clair que le scripteur s'implique dans les écrits, soit en s'alliant à son lectorat (on peut alors retenir ; on voit que ; on ne pouvait ignorer), soit en s'incluant dans la communauté d'enseignants praticiens (on simplifiera les écrits).

Une seule fiche de lecture propose un effacement total avec reprise de la voix de l'auteur, c'est-àdire qu'on ne voit non seulement aucun « je », « nous », « on » mais, de plus, aucune formule du type l'auteur dit que. Cet effacement total est rendu possible par le format de la fiche de lecture avec le référencement de l'ouvrage en première partie de la fiche de l'auteur ainsi que la séparation entre les trois parties de la fiche.

4.4 Copier-coller

Afin d'identifier les éventuels plagiats dans les fiches de lecture, nous nous sommes appuyée sur deux logiciels anti-plagiat qui permettent de relever le taux de similitude entre le document remis et des ressources Internet ${ }^{12}$. Il est couramment admis que si le logiciel détecte un taux de similitude supérieur à $10 \%$, il y a de grandes chances que l'étudiant se soit appuyé sur des écrits faisant partie de la base de données du logiciel. Or, avec un taux de similitude très faible de l'ordre de $0.4 \%$ pour l'ensemble des fiches de lecture, nous pouvons avancer que les étudiants n'ont pas fait de copier-coller ${ }^{13}$. Ceci confirme par la même occasion que les pavés citationnels ont peu été utilisés car ils sont facilement repérés dans des analyses anti-plagiat. Ce résultat reflète peut-être l'enseignement en amont qui a permis aux étudiants de repérer les différents types de plagiat à éviter et les façons de citer.

\section{Commentaires}

\subsection{Position du scripteur}

\footnotetext{
${ }^{12}$ A l'heure actuelle, peu de livres sont numérisés et disponibles en ligne. En conséquence, les comparaisons sont principalement effectuées entre le document soumis et des sites Internet (site de ventes avec quatrième de couverture ou court descriptif d'un ouvrage ; revues en ligne ; synthèses ou présentations en ligne de chercheurs ; sites thématiques, par exemple Eduscol...). Il est donc difficile de pouvoir s'assurer qu'un étudiant n'a pas copié des parties d'un livre ou repris des parties de documents déjà soumis à un autre enseignant.

${ }^{13}$ Cette affirmation peut être modulée en précisant que les étudiants n'ont pas fait de copier-coller de partie de sites Internet.
} 
Dans la seconde partie de la fiche de lecture, les étudiants devaient se positionner par rapport aux écrits. Pour ce faire, 15 étudiants ont utilisés le «je» ou le «nous» pour assumer leur point de vue (ex : J'ai trouvé intéressant; nous pouvons constater). Dans les autres cas, les étudiants s'appuient sur les structures Cet ouvrage..., ce livre..., cet article... et la formule L'auteur... est très peu présente alors qu'elle abondait dans la partie résumé de la fiche de lecture. En général, les commentaires sont courts (quelques lignes) et trois fois moins longs que les résumés. L'essentiel des commentaires repose surtout sur des impressions générales portant sur le contenu, l'organisation, la pertinence par rapport au thème de recherche... (ex : le livre est intéressant. Il dresse un tableau complet des problématiques. Incontournable, complet, pratique... $)^{14}$. D'ailleurs, les commentaires de 12 fiches de lecture ne contiennent que des impressions générales; un des commentaires est hors-sujet et 2 fiches de lecture reposent sur des applications éventuelles des écrits théoriques.

Six fiches de lecture questionnent le fond du texte lu. Les marqueurs de désaccord sont peu présents (on peut cependant remettre en question, il est dommage que, l'auteur ne cite pas) et ils sont amenés avec beaucoup de diplomatie (...mais peut-être manque-t-il d'un peu d'objectivité ?, Sauf erreur de notre part, cet article ne mentionne nulle part un apprentissage centré sur l'apprenant). Cette manière de ne pas questionner le texte est typique des étudiants qui entrent dans le champ de la recherche. On citera de Pollet et Piette (2002:176) le «moi, je suis étudiant, je n'ai pas le droit de critiquer !», la peur de parler en son nom propre (Rinck, 2006) et la difficulté à développer un point de vue (Delcambre et Reuter, 2002).

Si l'étudiant a du mal à se positionner comme constructeur de savoirs, il peine encore plus pour établir un dialogue. Seules 5 fiches de lecture établissent des liens avec d'autres citations ou d'autres concepts. Il s'agit par exemple de comparer un texte avec une autre fiche de lecture (Cet article s'accorde bien avec les recherches de Christine Hélot), de mettre le texte en regard avec d'autres textes (Un article qui brille par sa pertinence, mais qui doit néanmoins encore explorer plus en profondeur ce qu'une autre auteure, Haydée Silva, appelle dans son ouvrage "le jeu en classe de langue", les quatre régions métaphoriques de la notion de jeu (p.14) à savoir, le matériel ludique, les structures ludiques, le contexte ludique et l'attitude ludique...), d'intégrer des questions (je me questionne quant à la place et l'importance de l'identité dans les deux dynamiques linguistiques étudiées) ou de questionner/confirmer des citations et les notions-clés: (J'ai aussi remarqué au cours de mes lectures et cet ouvrage ne fait que le confirmer que le plurilinguisme est indissociable du pluriculturalisme et donc de l'interculturel).

La partie commentaires était censée forcer les étudiants à prendre position par rapport à leurs lectures, mais force est de constater que la plupart des points de vue développés sont affectifs et subjectifs, observations déjà consignées par Delcambre (2001) lors d'une étude sur des fiches de lecture avec une partie de commentaire de quatre pages. Alors que « la distance par rapport aux sources est fondamentale pour l'établissement d'un dialogue fructueux qui permettra l'émergence d'une problématique et d'éléments de résolution» (Pollet et Piette, 2002: 176), l'étudiant débutant une formation en recherche n'arrive pas à mettre en synergie ses lectures pour construire un début de questionnement.

\footnotetext{
${ }^{14}$ Un exemple complet de commentaire : Cet article est intéressant car l'auteur nous expose la possibilité d'adapter différentes stratégies d'apprentissage des langues étrangères envers des apprenants de niveau débutant en les mélangeant dans une même classe que leur langue maternelle soir proche ou lointaine de la langue cible.
} 


\subsection{Articuler résumé et commentaires}

Il est à noter que les étudiants n'utilisent aucun lien formel (connecteurs, reprises d'énoncés, expressions de transitions) pour relier la partie résumé à la partie commentaires.

En revanche, il est possible en observant de plus près ces deux parties de définir des profils types de fiches de lecture de la plus élémentaire à la plus dialogique. Le tableau ci-dessous résume la répartition de ces formats ${ }^{15}$ :

\begin{tabular}{|l|l|c|}
\hline \multicolumn{1}{|c|}{ Résumé } & \multicolumn{1}{c|}{ Commentaires } & \multicolumn{1}{c|}{ Nombre } \\
\hline Présentation chronologique/juxtaposition & Généraux & 10 (6 non-francophones) \\
\hline Présentation chronologique/juxtaposition & Point de vue & 4 \\
\hline Présentation chronologique/juxtaposition & Dialogique & 2 \\
\hline Présentation thématique & Généraux & 2 \\
\hline Présentation thématique & Point de vue & 1 \\
\hline Présentation thématique & Dialogique & 4 \\
\hline
\end{tabular}

La plupart des fiches suivent un format simple (résumé chronologie/commentaires généraux) et une seule mobilise un résumé thématique avec un commentaire dialogique. Nous remarquons aussi que les 6 locuteurs non-francophones ont suivi le format du résumé chronologique ; les fiches plus «élaborées » semblent donc plus difficilement réalisables par cette population. On remarque aussi que la fiche la plus aboutie fait partie d'un lot de 5 fiches qui étaient précédées par une page de questionnement initial et qui donnait déjà une orientation de lecture. Une problématisation en amont permet peut-être de mieux repérer et questionner les notions-clés.

\section{La fiche de lecture comme écrit de transition et de formation}

Nos propositions didactiques s'inscrivent dans le dispositif de formation aux écrits de recherche que nous avons décrit au début de notre propos. La fiche de lecture apparait comme un exercice pertinent pour l'acculturation aux écrits universitaires. Cependant, notre analyse montre les limites d'une approche «brute » de cet exercice qui mérite un accompagnement ciblé sur plusieurs points. Tout d'abord, une présentation des aspects formels de la citation (îlots citationnels, citation autonome) et des guillemets s'avère nécessaire. Un travail sur les verbes introducteurs pour la citation et la reformulation permettrait aussi de varier les textes. Les fonctions de la citation peuvent aussi être abordées sachant que dans le cadre de la partie résumé d'une fiche de lecture, la fonction principale de la citation est d'asseoir des définitions. Finalement, un exercice de relecture et de correction par des pairs d'une fiche de lecture avec une mise en commun des difficultés récurrentes devrait permettre d'éliminer la plupart des problèmes d'écriture que nous avons notés pour la première partie de la fiche de lecture.

Les étudiants doivent aussi prendre conscience de l'importance des commentaires pour engager un dialogue avec leurs lectures. A cet effet, une typologie des commentaires telle que proposée par Delcambre (2001 : 141-142) ${ }^{16}$ pourrait constituer un point de réflexion. Les commentaires doivent garder une fonction heuristique qui permet de construire l'objet d'étude et de commencer à

\footnotetext{
${ }^{15}$ Pour rappel, trois fiches étaient hors sujet

${ }^{16}$ a) impossible décollage ; b) exploration en franc-tireur ; c) élève appliqué ; d) distance et intégration maximales
} 
organiser son argumentation. Guibert (2001) propose des ateliers d'écriture afin de travailler sur des écrits intermédiaires en groupe et de faire émerger l'écriture-discussion et l'écriture polémique. Ceci éviterait aussi peut-être le phénomène de sous-énonciation (Rabatel, 2002) qui se retrouve dans les travaux du cours suivant intitulé «Définition d'une problématique ». Pour faire le lien avec ce cours, les fiches de lecture pourraient être plus situées en proposant aux étudiants de rédiger dans un premier temps une problématique naïve de leur thème de recherche et de la retravailler au vu de leurs lectures.

Au final, il s'agit d'amener les étudiants à mieux saisir les différents niveaux des macrocompétences de la production écrite tels que décrits par Mangiante et Parpette (2011:161) pour des apprenants allophones. Le premier niveau de compétence correspond à reproduire, identifier et recopier (par ex. prise de notes de textes brutes) ; le second niveau vise à apprendre à reformuler, étendre, diversifier (par ex. citation, reformulation); le troisième niveau de compétence porte sur relier, apparier, créer (par ex. développer les connexions entre différentes parties, différents textes, différents contextes). On voit ici que cette gradation des compétences de production écrite s'inscrit pleinement dans le déroulé d'une fiche de lecture comme exercice pour développer des compétences en littéracies universitaires.

\section{Conclusion}

Notre étude montre combien il est important de travailler sur des écrits de transition/d'étayage pour comprendre les stratégies mobilisées dans des écrits longs de recherche en formation (mémoires). En effet, cette recherche nous informe sur le répertoire réduit des étudiants quand vient le temps de la citation et du commentaire dans un contexte relativement restreint (la fiche de lecture). Les propositions pédagogiques qui élargissent le répertoire des stratégies et pointent les liens forme/fonction doivent s'appuyer autant sur les recherches en littéracies universitaires que sur celles en FOU afin de privilégier l'écriture universitaire (processus) pour développer des écrits (productions) plus en phase avec les exigences du monde scientifique.

\section{Bibliographie}

BLASER, C., ERPELDING-DUPUIS, P., 2010, « Cours d'appropriation des écrits universitaires: de l'analyse des besoins à la mise en œuvre, dans C. Blaser, M.-C. Pollet (dir.), L'appropriation des écrits universitaires, Diptyque, 18

< http://www.forumlecture.ch/blaser_2011_1.cfm>

BOCH, F., GROSSMANN, F., 2002, "Se référer au discours d'autrui : comparaison entre experts et néophytes », Enjeux, 54, p. 41-51.

BOCH, F. GROSSMANN, F., (dir.), 2001, Apprendre à citer le discours d'autrui, Lidil, 24, Grenoble, ELLUG.

BERTOCCHINI, P., COSTANZO, E., 2008, Manuel de formation pratique pour le professeur de FLE, Paris, Clé international.

CUQ, J.-P., 2003, Dictionnaire de didactique du français langue étrangère et seconde, Paris, Clé international.

CUQ, J.-P., GRUCA, I., 2002, Cours de didactique du FLES. Grenoble, PUG.

DELAMOTTE-LEGRAND, R., 2002, «Faire savoir son savoir : Polyphonie et mise en débat d'idées dans des copies d'étudiants », Enjeux, 54, p. 28-40. 
DELCAMBRE, I., 2001, «Formes diverses d'articulation entre discours d'autrui et discours propres : analyse de commentaires de textes théoriques », Lidil, 24, p. 135-166.

DELCAMBRE, I., REUTER, Y., 2002, «Images du scripteur et rapport à l'écriture », Pratiques, 113-114, p. 7-28.

FLØTTUM, K., JONASSON, K., NOREN, C., 2007, ON. Pronom à facettes, Bruxelles, DuculotDe Boeck.

FLØTTUM, K., THUE VOLD, E., 2010, «L'ethos auto-attribué d'auteurs-doctorants dans le discours scientifique », Lidil, 41, p. 41-58.

GUIBERT, R., 2001, «Citer et se situer : l'apprentissage de 1'écriture avec les discours d'autrui », Lidil, 24, p. 29-48.

GUIGUE, M., 1998, «Lectures et données de terrain. Deux modes d'accès au savoir difficiles à tisser dans la rédaction d'écrits longs », Lidil, 10, p. 81-95.

KARA, M., 2004, «Pratiques de la citation dans les mémoires de maîtrise », Pratiques, 121-122, p. 111-142.

MANGIANTE, J.-M., PARPETTE, Ch., 2004, Le français sur objectifs spécifiques : de l'analyse des besoins à l'élaboration d'un cours, Paris, Hachette Fle.

MANGIANTE, J.-M., PARPETTE, Ch., 2011, Le français sur objectifs universitaires, Grenoble, Presses universitaires de Grenoble.

POLLET, M.-C. 2001. Pour une didactique des discours universitaires : Etudiants et système de communications à l'université, Bruxelles, De Boeck Université.

POLlET, M.-C., PIETTE, V., 2002, «Citations, reformulations du discours d'autrui : une clé pour enseigner l'écriture de recherche ? », Spirale, 29, p. 165-179.

RABATEL, A., 2002, «Le sous-énonciateur dans les montages citationnels », Enjeux, 54, p. 5266.

ROBERT, J.-P., 2008, Dictionnaire pratique de didactique du FLE, Paris, Ophrys.

REUTER, Y., 2001, «Je suis comme un autrui qui doute. Le discours des autres dans l'écrit de recherche en formation », Lidil. 24, p. 13-28.

REUTER, Y., 2004, "Analyser les problèmes de l'écriture de recherche en formation », Pratiques, 121-122, p. 9-27.

REUTNER, U., 2010, «De nobis ipsis silemus? Les marques de personne dans 1'article scientifique », Lidil, 41, p. 79-102.

RINCK, F., 2006, «Gestion de la polyphonie et figure de l'auteur dans les parties théoriques de rapports de stage », Lidil, 34, p. 85-103.

RINCK F., TUTIN A., 2007, «Annoter la polyphonie dans les textes : le cas des passages entre guillemets », Corpus, 6, p. 79-100.

TARDIEU, C., 2008, La didactique des langues en 4 mots-clés : communication, culture, méthodologie, évaluation, Paris, Ellipses.

TUTIN, A., 2010, «Dans cet article, nous souhaitons montrer que... lexique verbal et positionnement de l'auteur dans les articles en sciences humaines », Lidil, 41, p. 15-40. 


\section{Annexe 1. Consignes}

Fiches de lecture - Format

\section{- Référence}

- Type de document

- Auteur

- Année

- Titre

- Périodique

- Lieu de publication

- Editeur

- Nombre de pages

- ISSN

- Mots-clés

- Fiche établie par (Nom, Prénom) le (date)

- Résumé (avec ou sans citations)

- Commentaires 


\section{Annexe 2. Fiche modèle proposée par un étudiant}

\section{Références}

Type de document : périodique

Auteur : Puren, Christian

Année : 2001

Titre : «Représentation de l'enseignement / apprentissage de la grammaire en didactique des langues.»

Périodique : Ela. Etudes linguistique appliquée

Lieu de publication : Paris

Editeur : Klincksieck

Nombre de pages : 8

ISSN : 0071-190X

Mots-clés : grammaire, enseignement, apprentissage, didactique, langues

Fiche établie par Zell, Laetitia, le 19 Octobre 2012

\section{Résumé}

Christian Puren décrit la représentation de l'enseignement et de l'apprentissage de la grammaire en didactique des langues. Selon lui, on peut distinguer 4 représentations (modèles) du rapport entre grammaire et didactique :

- Modèle 1 = langue-objet/enseignement-objet où la grammaire est un outil qui sert à décrire le fonctionnement de la langue (objet). L'enseignement est centré et basé sur la grammaire avec des supports préfabriqués. C'est pourquoi ce modèle/rapport met les enseignants natifs et non natifs à égalité, puisqu'il repose sur le savoir académique de la langue.

- Modèle 2 = langue-objet / apprentissage-objet où la langue, comme dans le modèle précédent est toujours un objet mais c'est l'apprenant, qui utilise l'outil grammaire pour planifier c'est-à-dire que l'apprenant est acteur et fait progresser son apprentissage, selon son rythme et ses besoins pour une meilleure appropriation.

- Modèle 3 = langue-objet/enseignement-sujet apprenant où Puren explique le concept de démarche active en grammaire. Si les élèves emploient une démarche inductive pour conceptualiser la grammaire, celle-ci est incomplète puisque l'enseignant reste le référent suprême, gardien du savoir qui a le contrôle sur la façon de procéder de ses apprenants et notamment «l'énoncé des règles « standard » telles qu'elles apparaissent dans sa grammaire de référence » (p.5).

- Modèle 4 = langue-sujet natif/enseignement-sujet apprenant où l'enseignement de la grammaire est basé sur les intentions énonciatives de locuteurs natifs dans des documents authentiques. Les apprenants, employant toujours une démarche inductive, s'approprient alors le langage des locuteurs natifs ainsi que son fonctionnement, de manière intuitive. Ce modèle favorise les enseignants natifs à cause de la langue de communication et des documents authentiques utilisés, recelant des implicites et intentions.

Christian Puren termine son article en établissant une cinquième représentation : languesujet/apprentissage-sujet où le processus d'appropriation est vu comme une succession de procédés cognitifs, propres à chaque apprenant mais aussi à chaque contexte. Ainsi, l'apprentissage est variable d'un individu à l'autre mais également d'un contexte à l'autre. 
Puren conclut donc en disant qu'il est impossible d'établir une « théorie de l'enseignement/ apprentissage des langues » puisque la didactique est une « discipline d'observation et d'intervention » (p.6); il s'agit donc d'une modélisation et non d'une théorisation qui propose des outils aux enseignants : «il ne s'agit plus de décrire le processus d'enseignement, ni le processus d'apprentissage, mais d'observer et de gérer en contexte les phénomènes complexes de contact entre ces deux processus. »(p.7)

\section{Commentaires}

L'article est intéressant car il montre l'évolution de la représentation et de l'enseignement de la grammaire au fil des méthodologies (centration sur la grammaire (et donc enseignant référent, au sommet du triangle didactique) vers centration sur l'apprenant)

Aussi, par la description des différentes représentations, Puren convie les lecteurs, en l'occurrence les enseignants à prendre connaissance des différents modèles, de s'en inspirer ou non...

Il y décrit bien la didactique des langues qui ne peut se limiter à une théorisation mais qui est une discipline complexe, car sujette au contexte et il est bien expliqué qu'il est impossible d'obtenir une modèle de référence unique.

Cependant, cet article m'a paru bien théorique lors de la lecture : il manque d'exemples concrets, de situation d'enseignement et de contextualisation dans la vie réelle. Puren, à de multiples occasions, renvoie le lecteur à d'autres articles de la revue, qui pourraient éventuellement répondre à ses questions, ce qui est dommage car l'article ne semble pas aller en profondeur des choses et illustrer suffisamment les propos de l'auteur. 


\section{Annexe 3 : Grille d'analyse}

Le résumé

- S'effacer:

- Non-utilisation du je

- Utilisation du nous/on

- Présenter des concepts- clés/ des idées-clés

- Citer

- Nombre de guillemets/citations

- Ilot citationnel/citation autonome/

- Reformulation

- Formules impersonnelles

- Utilisation du passif

- Tournures du type «L'auteur dit que ...»

- Calque de la voix de l'auteur

- Copier/coller

- Présenter l'auteur

- Présenter le support

Le commentaire

- Assumer un point de vue

- Utilisation de « je »

- Donner ses impressions (j'aime/j'aime pas; pertinence)

- Questionner le texte (fond)

- Marqueurs d'accord et de désaccord

- Confrontation avec d'autres définitions

Articuler résumé et commentaire

- Transition/Formatage

- Connecteurs

- Reprises d'énoncés 